\title{
PERSPECTIVE
}

\section{Diversity is the question, not the answer}

\author{
Ashley Shade \\ Department of Microbiology and Molecular Genetics, Program in Ecology, Evolution, and Behavior, and DOE \\ Great Lakes Bioenergy Research Center, Michigan State University, East Lansing, MI, USA
}

\begin{abstract}
Local diversity (within-sample or alpha diversity) is often implicated as a cause of success or failure of a microbial community. However, the relationships between diversity and emergent properties of a community, such as its stability, productivity or invasibility, are much more nuanced. I argue that diversity without context provides limited insights into the mechanisms underpinning community patterns. I provide examples from traditional and microbial ecology to discuss common complications and assumptions about within-sample diversity that may prevent us from digging deeper into the more specific mechanisms underpinning community outcomes. I suggest that measurement of diversity should serve as a starting point for further inquiry of ecological mechanisms rather than an 'answer' to community outcomes.
\end{abstract}

The ISME Journal (2017) 11, 1-6; doi:10.1038/ismej.2016.118; published online 16 September 2016

'The rock just sits and is.' - Albert Markovski

Diversity (the number and/or evenness, and types of taxa within a local community) is arguably one of the most fundamental concepts in community ecology. Ecologists report diversity estimates for a number of reasons, including to ask how patterns in diversity translate to ecosystem function or stability, or to understand how and why diversity changes over space and time. This research is pursued in an effort to build an understanding of the ecological processes of the world and to identify common patterns, with the ultimate goal of improving knowledge of universal mechanisms and building theoretical framework. Once ecological mechanisms are understood, ecologists strive to better predict, conserve or manage communities to desired outcomes.

Microbial ecologists have a particular interest in diversity, as microbial diversity is expansive, and many microbial communities have nearinnumerable membership (Locey and Lennon, 2016). Relative to our planet's better-described communities of macrofauna, microbial communities are a trove of untapped and unknown diversity. High-throughput sequencing methods have offered new insight into the extent and limits of microbial diversity, and have spurred new research interests in diversity, including

Correspondence: A Shade, Department of Microbiology and Molecular Genetics, Program in Ecology, Evolution, and Behavior, and DOE Great Lakes Bioenergy Research Center, Michigan State University, 2215 Biomedical Physical Sciences Building, 567 Wilson Road, East Lansing, MI 48824, USA.

E-mail: shadeash@msu.edu

Received 23 May 2016; revised 7 July 2016; accepted 2 August 2016; published online 16 September 2016 understanding the rare microbial biosphere (Lynch and Neufeld, 2015) and microbial dark matter (Marcy et al., 2007). High-throughput sequencing combined with cultivation-independent methods continue to reveal a fuller tree of life, expanding our knowledge of evolution and of phylogenetic relationships in biology, which are dominated by microbial lineages (Hug et al., 2016).

With all of the discovery and excitement in microbial ecology about diversity, there often has been an assumption that high diversity is implicitly a good or desirable outcome for communities, and that higher diversity is also somehow more meritorious ecologically. Alas, diversity is not good or bad, it simply 'is', much like Albert Markovski's rock. Diversity is a property that we observe about microbial communities and measure using statistical indices, and these measurements allows us to develop hypotheses to test the ecological mechanisms driving those communities' dynamics. Diversity is the outcome of ecological processes and not an ecological process in itself. Thus, a diversity measurement has limited value alone because much context is needed for interpretation. I argue that diversity provides a proxy for comparing communities, and an appropriate starting point for determining underlying ecological causes and consequences in community ecology. Similarly, I suggest that as a field, we resist the oversimplification of implicating diversity as a reason for community outcomes or implying that a high-diversity microbial community is somehow 'better' than a low-diversity community.

Here, I explore the complications and assumptions about microbial diversity and offer suggestions to redirect some of our common misconceptions toward understanding the ecological mechanisms 
Table 1 Different aspects of within-sample (alpha) diversity

\begin{tabular}{|c|c|}
\hline Aspect of diversity & Notes \\
\hline Community size & The total number of individuals observed in a locality \\
\hline Number of taxa (richness) & Summarizes the total number of taxa, where taxon is counted as an equivalent unit \\
\hline Equitability of taxa (evenness) & Summarizes how evenly distributed are relative contributions across taxa \\
\hline Composition of taxa & Accounts for the number of unique taxa and their identities, which can be taxonomic or operational \\
\hline Relative contributions of taxa & Accounts for the proportional contributions of each taxon to the total count of all individuals observed \\
\hline Phylogenetic relatedness of taxa & The evolutionary breadth represented by taxa given a phylogenetic tree \\
\hline
\end{tabular}

One or more of these aspects are incorporated into common diversity estimates, like Shannon diversity, Faith's phylogenetic diversity and so on.

driving patterns in diversity. I focus on withinsample (alpha) diversity of a locality (Table 1; see Magurran, 2003 for an excellent primer).

\section{Diversity has many definitions. Which are you using and why does it matter?}

There is a long history of challenge with both defining and measuring ecological diversity (for example, Ricotta, 2005). An inherent challenge is that there is no universally accepted, absolute value of diversity for a given community. Contrast this to other quantitative measurements considered to have absolutes that are comparable across different methods or scales of measuring. For example, whether temperature is measured in Kelvin, Celsius or Fahrenheit, there is a belief that there is one 'true' equivalent value upon which all scales will agree. Diversity, however, is relative and always constrained by method of measurement. There is not a belief that an absolute diversity value can be determined and compared across methods: each method is a slightly different reduction of multivariate information about a community. If determining an absolute value of diversity were achievable (and it likely is not), it would unite the field of community ecology by ending the debate about the merits of different diversity measurements, and instead redirecting focus toward underlying mechanisms.

Thus, 'diversity' can refer to be any number of metrics considering any one or number of aspects of a community. Because no absolute value of diversity exists, each method has its own biases and advantages, as discussed previously (for example, Hill et al., 2003). A lack of specificity about which method is used can lead, at best, to confusion, or at worst, oversimplification or misinterpretation of community outcomes. Thus, the precise method(s) for calculating diversity should be carefully considered, and justified either ecologically or biologically according to the scientific question, and then interpreted considering the chosen diversity metric's strengths and limitations. For example, if there is a working hypothesis of differences in phylogenetic breadth between communities in a control and treatment, a diversity metric that incorporates information about the relatedness of taxa may be selected.

Diversity metrics are, by design, flexible, and thus can be calculated from any type of community data set. Because it is difficult to observe individual microbial cells and distinguish among microbial taxa, microbial ecologists use many inexact methods for observing communities, including cell morphology or probe binding with microscope counts, fingerprinting, colony phenotypes, and sequencing. Each of these methods produces a differently biased perspective of the community. Thus, owing to methodological differences, diversity often is not comparable directly across studies even if the same metric is calculated. This results in vagueness and does not promote a deeper understanding of microbial community ecology. It also means that diversity cannot be 'important' in itself because much context is needed for interpretation.

\section{The temptation of diversity: it is easy to calculate, but let us not forget the limitations}

For a high-throughput sequencing data set, diversity is straightforward to determine, perhaps in part, because popular sequence analysis pipelines automatically output these calculations. The ease of diversity calculation may tempt us to indiscriminately report them or to assume that they are informative for our study. However, for sequencing methods, there are a range of analysis choices regarding the operational taxonomic unit definition (Eren et al., 2013; Preheim et al., 2013; Rideout et al., 2014; Schloss, 2016). The taxonomic unit is whatever is appropriate to the scientific question (or, as it often happens, default in the sequence analysis pipeline), as chosen by the researcher. This is an important consideration because operational taxonomic unit definitions will impact our perspective of diversity, and some operational taxonomic unit-defining methods consistently over or underinflate the number of taxa observed (for example, Edgar, 2013), which directly impacts diversity calculations.

There are also inherent diversity biases resulting from cultivation-independent methods. Diversity 
metrics may be inflated by the DNA of inactive or dormant organisms (Jones and Lennon, 2010). DNA extraction protocols can bias against lyses of certain groups, skewing their representation in the community (for example, Gram-positive bacteria). Coextraction of relic or taphonomic DNA can overestimate standing diversity (Carini et al., 2016). There is also primer bias in amplicon sequencing, which can underestimate diversity by omitting or underrepresenting certain microbial lineages (Klindworth et al., 2013). For some marker genes, like the $16 \mathrm{~S}$ ribosomal RNA gene, bacterial and archaeal taxa may have very different copy numbers (Stoddard et al., 2015), which complicates our perception of their relative contributions to the community. For taxon-rich microbial communities, like soils, and especially for highly uneven, rich communities that are dominated by a few very abundant taxa (Adams et al., 2013), undersampling of the community is an additional consideration (for example, Gihring et al., 2012). Increasing the amount of sequences generated for undersampled communities resulted in continued increase in diversity estimates, with particular sensitivity in the performance of nonparametric estimators that extrapolate absolute community diversity based on the number of singletons and doubletons (Gihring et al., 2012).

Of course, no method is without bias. However, owing to our inexact methods for observing communities, microbial diversity calculations in particular have so many biases that they may be considered as rough approximations. Efforts should be made to standardize biases across samples prior to making diversity comparisons, and, even then, to interpret results with care.

\section{High diversity is not necessarily 'better' or 'healthy'}

If higher diversity were universally better for communities, why devote resources to understanding ecological mechanisms? If it were true that higher diversity is always an improvement, we could manage microbial communities by simply making them more diverse.

There are countless examples of ecosystems in which higher diversity is not more meritorious. As a simple example, a rainforest harbors more plant species per hectare than a temperate forest, but it is not interpreted that the temperate forest is a less important or less-thriving ecosystem. These two ecosystems are different, and for many abiotic or biotic reasons that could be uncovered and investigated further. Similarly, vaginal microbial communities exhibit a range of diversities across healthy women, including some communities that are dominated by lactobacilli and others (reported as $20 \%-30 \%$ of asymptomatic individuals) that have less lactobacilli but more diverse membership (Ma et al., 2012). As another example, high-fat and low-fat diets had comparable levels of Shannon diversity, Chao diversity and richness (though, different taxonomic compositions) in humanized mouse models (Turnbaugh et al., 2009). These studies and others demonstrate that lower diversity is not necessarily indicative of a worse community or ecosystem, and lower diversity does not necessarily imply less stable or less 'healthy' communities.

There is a recent example in which we do overwhelm microbial communities with more and different diversity in an effort to manage them: fecal transplants after an opportunistic infection by Clostridium difficile (Kassam et al., 2013). Not all fecal transplants are successful, despite the apparent deluge of additional diversity to the community. It would be a misinterpretation to suggest that the diversity in itself is the answer to the frequent success of fecal transplants in mitigating $C$. difficile infection.

Notably, microbial ecologists have borrowed value-laden terms from traditional ecology to describe diversity, which may be one historical reason for the persistence of assumptions that higher diversity is better. For example, ecologists use richness to refer to the number of species, and depauperate to describe communities with low diversity. This terminology was used in the literature as early as the 1920's (for example, Wheeler, 1926), but seems to have become more prominent by the 1940's (for example, Hubbs and Lagler, 1949). Thus, microbial ecologists perhaps have intuited value from legacy jargon without reconsideration of its merit.

\section{Diversity only has value in a comparative context}

Except in the context of informing study design and approach (for example, how many sequences are needed for exhaustive coverage of a community?), there is little ecological value in reporting that a community has 10000 taxa or 10 . The insight emerges when comparing that community with another situation or community of interest, and then asking what is the difference observed and why. This could be in the context of an experimental design between control and treatment conditions, over a natural or controlled environmental gradient, over time, in response to a disturbance or stressor, or over geographic space.

Comparative microbial diversity, especially when multilayered community and functional measurements are applied, has provided key insights into underlying processes. For example, two sets of replicated methanogenic bioreactors responded differently to a pulse glucose shock: one set was stable, whereas the other decreased in performance (Fernandez et al., 2000; Hashsham et al., 2000). It was discovered that the sets harbored very different microbial compositions, measured using several 
complementary methods, including cell morphology, fingerprinting and ribosomal RNA probes, which allowed the researchers to delve more precisely into the comparative mechanisms of functional stability. In particular, the bioreactors with less diverse membership had an ability to perform parallel substrate processing during the glucose shock to maintain performance. One important conclusion from this study was that communities with higher diversity were not necessarily more functionally stable in the face of disturbance.

Because of all of the nuances in calculating diversity, comparing diversity within a single study or across a series of related studies (often by the same researcher) provides situation-specific insights and modest advances. For understanding larger scale patterns in diversity over space or time, or across many ecosystems, researchers often have to spend much time curating disparate data sets for metaanalysis, and redefining taxonomic units across studies to be maximally comparable. Even then, owing to methodological differences, each data set sometimes must remain as a distinct unit and quantitative cross-study comparisons are limited. Though quantifying large-scale patterns in microbial ecology is challenging, one of the scientific reasons for doing so is to test ecological theories established in traditional ecology for microbial communities. Calculating diversity for microbial communities and analyzing their overarching patterns using methods directly comparable to studies in traditional ecology pushes forward our pursuit of a unified ecological theory. For example, studies have considered latitudinal gradients of diversity (for example, Chu et al., 2010), and species-area and species-time relationships (for example, Bell et al., 2005; Shade et al., 2013). In some cases, microbial diversity exhibits similar large-scale patterns to communities of larger organisms ('macroorganisms', for example, Locey and Lennon, 2016), and in some cases, they are distinct (for example, Fierer et al., 2011). Understanding these points of distinction for microbial diversity will allow us to delve deeper into the ecological mechanisms driving their patterns, and better place them in the context of a grander view of biology. To provide specific example of how comparisons of large-scale patterns in diversity can uncover common underlying ecology, a recent meta-analysis used species richness, observed community size, and maximum community size of both macrobial and microbial communities to discover a universal scaling law relationship between the size of the community and its evenness (inclusive of dominance and rarity), where larger communities exhibit lower evenness and a larger 'rare biosphere' (Locey and Lennon, 2016).

A final consideration is whether a given diversity comparison is ecologically meaningful. For example, can any insight be gleaned to consider that an acid mine drainage community is much less diverse than a soil (or, to be a bit facetious, that a mammal gut has different diversity than the surface of a kitchen counter)? In these examples, there are very distinct ecosystems with fundamentally different drivers and constraints. We do not need to calculate diversity in each to be led to hypotheses as to why they are different. An exception to this is in questions concerning source tracking of specific community members (for example, Knights et al., 2011), where disparate but connected ecosystems or regional metacommunities may be implicated in seeding each other's diversity.

\section{If diversity is not the answer, what is?}

There are many ecological mechanisms that underpin patterns in community diversity, and they are inherently difficult to unravel. The most commonly studied mechanisms are deterministic processes. For instance, abiotic drivers and constraints, like environmental filters and carrying capacity of an ecosystem, limit the type and number of organisms capable of exploiting the habitat. Abiotic disturbances may disrupt resource availability and make new niche space available, driving replacement or proportional changes in communities. Disturbances sometimes impact specific members rather than the whole community, driving selection, or release from competition or predation. Biotic interactions are also important drivers of diversity, and the nature and strength of interactions like antagonism and synergism can result in complex and nonintuitive multimember interactions (for example, Tilman, 1994). Thus, our ultimate understanding of diversity requires more than measurements of diversity; we also need contextual data and sufficient numbers of community observations for thoughtful comparisons that test specific hypotheses about how diversity may, or may not, change across sample categories or gradients.

Suppose a hypothetical study used marker gene sequencing to uncover evidence that a certain pathogen is more successful in invading a hostassociated microbial community that has relatively lower diversity as compared with a higher-diversity community. The researchers may then report that high diversity prevents pathogen invasion, and then attribute this to an underlying higher functional diversity in that community.

The question to consider is: what about the ecology of the more diverse community that is inhibitory toward the pathogen, and what about the less diverse community that is permissive? Perhaps, it is that there is a direct competitor of the pathogen in the more diverse community. Or, perhaps there is a mutualist of the pathogen in the low-diversity community that promotes its growth. Perhaps, the higher-diversity community has lower $\mathrm{pH}$, and the pathogen is sensitive to this specific abiotic driver. Perhaps, it is because a subset of community members has stimulated the host immune response in the higher-diversity community. Perhaps, it is 
because the higher-diversity community is at carrying capacity, and there are no available niches for the invading pathogen. Perhaps, the pathogen acquired a beneficial gene, via horizontal gene transfer, from a member of the lower-diversity community that improved its success. (Also, without directly measuring function or functional potential, it is a conceptual leap to move from the observation of high compositional diversity to the assumption of high functional diversity.)

The mechanisms maintaining or changing microbial diversity are many and complex. Understanding how these mechanisms collectively contribute to community outcomes is of great importance for the goals of predicting, conserving and managing microbial communities, and reporting diversity without underlying hypotheses, contextual data for interpretation or useful comparisons does not advance our understanding toward these goals. Furthermore, common assumptions that 'higher diversity is better' oversimplifies complex mechanisms and can sidetrack progress. There is a lot of work to be carried out, and measuring diversity is the first step in a rich line of scientific inquiry. Measurement of diversity should serve as a starting point for further inquiry of ecological mechanisms rather than an 'answer' to community outcomes.

\section{Conflict of Interest}

The author declare no conflict of interest.

\section{Acknowledgements}

This work was supported in part by Michigan State University, and in part by the DOE Great Lakes Bioenergy Research Center (DOE BER Office of Science DE-FC0207ER64494) and the DOE OBP Office of Energy Efficiency and Renewable Energy (DE-AC05-76RL01830). I thank Noah Fierer and Jackson Sorensen for the insightful discussions, and reviewers for valuable comments on the work.

\section{References}

Adams RI, Amend AS, Taylor JW, Bruns TD. (2013). A unique signal distorts the perception of species richness and composition in high-throughput sequencing surveys of microbial communities: a case study of fungi in indoor dust. Microb Ecol 66: $735-741$.

Bell T, Ager D, Song JI, Newman JA, Thompson IP, Lilley AK et al. (2005). Larger islands house more bacterial taxa. Science 308: 1884.

Carini P, Marsden PJ, Leff JW, Morgan EE, Strickland MS, Fierer N. (2016). Relic DNA is abundance in soil and obscures estimates of soil microbial diversity. PeerJ Prepr. http://dx.doi.org/10.1101/043372.

Chu H, Fierer N, Lauber CL, Caporaso JG, Knight R, Grogan P. (2010). Soil bacterial diversity in the Arctic is not fundamentally different from that found in other biomes. Environ Microbiol 12: 2998-3006.

Edgar RC. (2013). UPARSE: highly accurate OTU sequences from microbial amplicon reads. Nat Methods 10: $996-998$.

Eren AM, Maignien L, Sul WJ, Murphy LG, Grim SL, Morrison HG et al. (2013). Oligotyping: differentiating between closely related microbial taxa using $16 \mathrm{~S}$ rRNA gene data. Methods Ecol Evol 4: 1111-1119.

Fernandez AS, Hashsham SA, Dollhopf SL, Raskin L, Glagoleva O, Dazzo FB et al. (2000). Flexible community structure correlates with stable community function in methanogenic bioreactor communities perturbed by glucose. Appl Environ Microbiol 66: 4058-4067.

Fierer N, McCain CM, Meir P, Zimmermann M, Rapp JM, Silman MR et al. (2011). Microbes do not follow the elevational diversity patterns of plants and animals. Ecology 92: 797-804.

Gihring TM, Green SJ, Schadt CW. (2012). Massively parallel rRNA gene sequencing exacerbates the potential for biased community diversity comparisons due to variable library sizes. Environ Microbiol 14: 285-290.

Hashsham SA, Fernandez AS, Dollhopf SL, Dazzo FB, Hickey RF, Tiedje JM et al. (2000). Parallel processing of substrate correlates with greater functional stability in methanogenic bioreactor communities perturbed by glucose. Appl Environ Microbiol 66: 4050-4057.

Hill TCJ, Walsh KA, Harris JA, Moffett BF. (2003). Using ecological diversity measures with bacterial communities. FEMS Microbiol Ecol 43: 1-11.

Hubbs CL, Lagler KF. (1949). Fishes of Isle Royale, Lake Superior, Michigan. Pap Mich Acad Sci Arts Lett 33: 73-133.

Hug LA, Baker BJ, Anantharaman K, Brown CT, Probst AJ, Castelle CJ et al. (2016). A new view of the tree of life. Nat Microbiol 1: 16048.

Jones SE, Lennon JT. (2010). Dormancy contributes to the maintenance of microbial diversity. Proc Natl Acad Sci USA 107: 5881.

Kassam Z, Lee CH, Yuan Y, Hunt RH. (2013). Fecal microbiota transplantation for Clostridium difficile infection: systematic review and meta-analysis. Am J Gastroenterol 108: 500-508.

Klindworth A, Pruesse E, Schweer T, Peplies J, Quast C, Horn $M$ et al. (2013). Evaluation of general 16S ribosomal RNA gene PCR primers for classical and next-generation sequencing-based diversity studies. Nucleic Acids Res 41: 1-11.

Knights D, Kuczynski J, Charlson ES, Zaneveld J, Mozer MC, Collman RG et al. (2011). Bayesian community-wide culture-independent microbial source tracking. Nat Methods 8: 761-763.

Locey KJ, Lennon JT. (2016). Scaling laws predict global microbial diversity. Proc Natl Acad Sci USA 113: 5970-5975.

Lynch MD, Neufeld JD. (2015). Ecology and exploration of the rare biosphere. Nat Rev Microbiol 13: 217-229.

Ma B, Forney LJ, Ravel J. (2012). Vaginal microbiome: rethinking health and disease. Annu Rev Microbiol 66: 371-389.

Magurran AE. Measuring Biological Diversity. WileyBlackwell: Oxford, UK, 2003.

Marcy Y, Ouverney C, Bik EM, Lösekann T, Ivanova N, Martin HG et al. (2007). Dissecting biological 'dark matter' with single-cell genetic analysis of rare and uncultivated TM7 microbes from the human mouth. Proc Natl Acad Sci USA 104: 11889-11894. 
Preheim SP, Perrotta AR, Martin-Platero AM, Gupta A, Alm EJ, Perrott AR et al. (2013). Distribution-based clustering: using ecology to refine the operational taxonomic unit. Appl Environ Microbiol 79: 6593-6603.

Ricotta C. (2005). Through the jungle of biological diversity. Acta Biotheor 53: 29-38.

Rideout JR, He Y, Navas-Molina JA, Walters WA, Ursell LK, Gibbons SM et al. (2014). Subsampled openreference clustering creates consistent, comprehensive OTU definitions and scales to billions of sequences. PeerJ 2: e545.

Schloss PD. (2016). Application of a database-independent approach to assess the quality of operational taxonomic unit picking methods. mSystems 1: e00027-16.

Shade A, Caporaso JG, Handelsman J, Knight R, Fierer N. (2013). A meta-analysis of changes in bacterial and archaeal communities with time. ISME J 7: 1493-1506.

Stoddard SF, Smith BJ, Hein R, Roller BR, Schmidt TM. (2015). rrnDB: improved tools for interpreting rRNA gene abundance in bacteria and archaea and a new foundation for future development. Nucleic Acids Res 43: D593-D598.
Tilman D. (1994). Competition and biodiversity in spatially structured habitats. Ecology 75: 2-16.

Turnbaugh PJ, Ridaura VK, Faith JJ, Rey FE, Knight R, Gordon JI. (2009). The effect of diet on the human gut microbiome: a metagenomic analysis in humanized gnotobiotic mice. Sci Transl Med 1: 6ra14.

Wheeler WM. (1926). Ants of the Balearic Islands. Folia Myrmecol Termit 1: 1-6.

(c) (i) (2) This work is licensed under a Creative Ay ${ }_{\mathrm{NC}} \mathrm{sA}$ Commons Attribution-NonCommercialShareAlike 4.0 International License. The images or other third party material in this article are included in the article's Creative Commons license, unless indicated otherwise in the credit line; if the material is not included under the Creative Commons license, users will need to obtain permission from the license holder to reproduce the material. To view a copy of this license, visit http:/creativecommons.org/ licenses/by-nc-sa/4.0/ 